\title{
Design of Dual-Mode Engine Flowpaths for Hypersonic Vehicles Using Reduced-Order Models
}

\author{
Sean M. Torrez, Derek J. Dalle*, James F. Driscoll ${ }^{\dagger}$ \\ University of Michigan, Ann Arbor, MI 48109
}

\begin{abstract}
Hypersonic vehicle flow path design must be approached as an entire system because of the interactions between overall thrust and moment on vehicle performance and stability. It is therefore difficult to design hypersonic vehicles using conventional design techniques such as component optimization. This study examines the design problem with a more unified approach, considering tradeoffs throughout the design space in order to consider what causes a vehicle to have acceptable performance throughout. Several combustor designs from the open literature are examined using a reduced-order model (ROM). Some design principles are proposed for placement of injectors and location of wall divergences and their angles. An automatic design framework suitable for multidisciplinary optimization (MDO) is presented. Previous models did not include a model for switching between ram-mode and scram-mode operation; the derivation of such a model also is presented.
\end{abstract}

\section{Introduction}

Scramjet and ramjet combustors differ from conventional gas turbine combustors because their geometric compression components can have fundamentally different performance depending on the thermodynamic and chemical characteristics of the incoming flow. ${ }^{1}$ This means that the central question is not just how much of the fuel burns, but where and how the fuel burns. ${ }^{2}$ The MASIV code $^{3}$ used here generates vehicle geometries based on design principles developed by the authors and other ideas found in the open literature. This allows us to rapidly create vehicle configurations and analyze the quality of the designs.

The MASIV design methodologies focus on creating vehicle geometries with performance that is acceptable over a wide range of conditions. Previous work ${ }^{3}$ has shown that a vehicle designed to have high inlet pressure recovery factor (PRF) at a single design point will be likely to have much poorer inlet performance for conditions near to, but slightly displaced from, the design point. This can cause rapid changes in thrust around the design point, which in turn creates vehicle performance and control problems.

${ }^{*}$ Graduate Research Assistant, Department of Aerospace Engineering, AIAA Student Member

${ }^{\dagger}$ Professor, Department of Aerospace Engineering, AIAA Fellow 


\section{Flowpath design}

First, some terminology is introduced. We define the internal flow geometry, which is approximated as 2-D with a certain depth (into the page) a "flowpath." We define flowpath plus the outer mold line of the vehicle including all 3-D geometry required and all control and lifting surfaces to be the "vehicle." Thus the term "flowpath design" refers to the list of parameter values that result in a particular flowpath geometry. Correspondingly, a "vehicle design" refers to the list of external vehicle parameters that yield a certain vehicle geometry. In principle these lists can be quite extensive.

As stated above, we have in general cast the design problem in terms of performance over a range of conditions. These type of aggregate performance measures lend themselves to designs optimized for more than one conditions, which is deemed important for airplanes whose mission trajectories are not immediately clear.

For example, Fig. 1 shows a very basic flowpath geometry. This was the one of the first flowpath geometries we tested. Thrust performance of this geometry is shown in Fig. 2a. The performance of this design suffers greatly if there is a small perturbation from the design point (in this case $M=8$ ). A better design was found in our prior work ${ }^{4}$ and the geometry based on this design is shown in Fig. 3. Its performance is shown in Fig. 2b, which allows us to compare the two designs of the previous study. Looking at Fig. 2c, which compares the normalized performance of both designs, we see that there is a non-smooth point in the performance of the simple flowpath at $M=7$ and another a $M=8$. If We specify instead an inlet optimized for a range of conditions, as is shown in the dashed line, we see that these performance problems can be moved out of the range of operation of the inlet (in this case $7<M<9$ ). In this simple example it is clear that a vehicle whose performance suffers so abruptly a short distance from the design point would have unacceptable flight performance and control characteristics. The rest of this paper will offer a method for generalizing the description of these undesirable behaviors to a multidimensional space and will examine some existing designs for their sensitivity to changes in operating point.

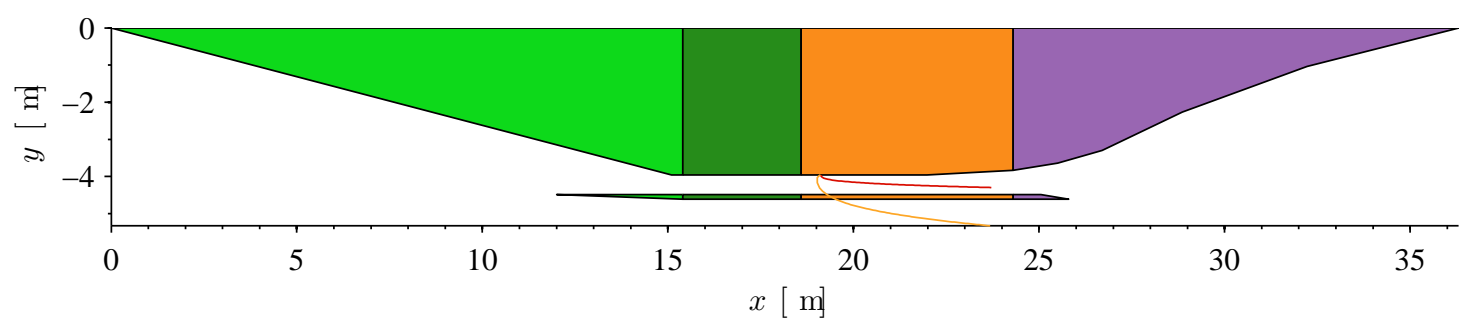

Figure 1. An example of a vehicle design that has peaky performance around the design point.

\section{Analysis of dual-mode combustors}

General 1-D flow solution for hypersonic flowpaths has been covered in the literature. ${ }^{5,6}$ However, since these results are obtained by solving a set of Ordinary Differential Equations (ODEs) they do not admit wave-type solutions. This difficulty becomes important if we wish to consider subsonic combustion cases, since the stability of the flow relies on the propagation of information upstream from the choking location in the combustor up to end of the supersonic inflow portion 


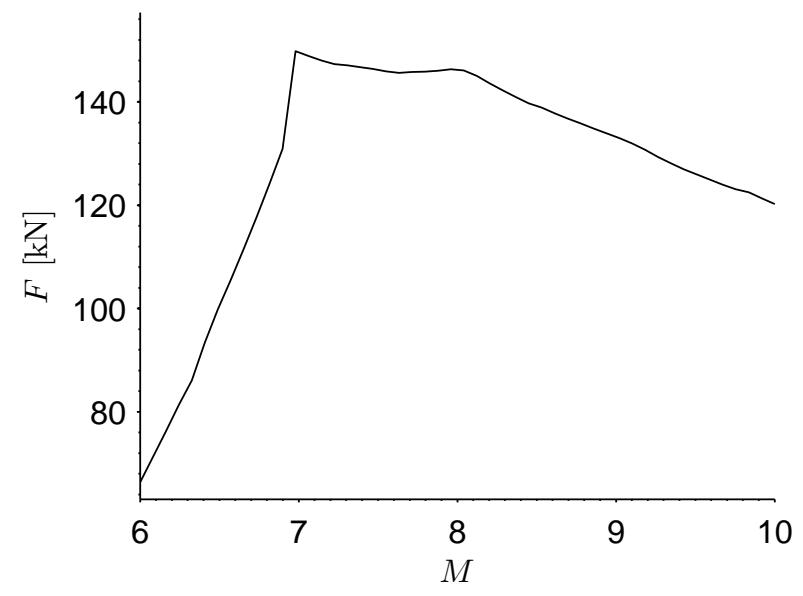

a) Thrust vs Mach number for the simple design in Fig. 1, in N.

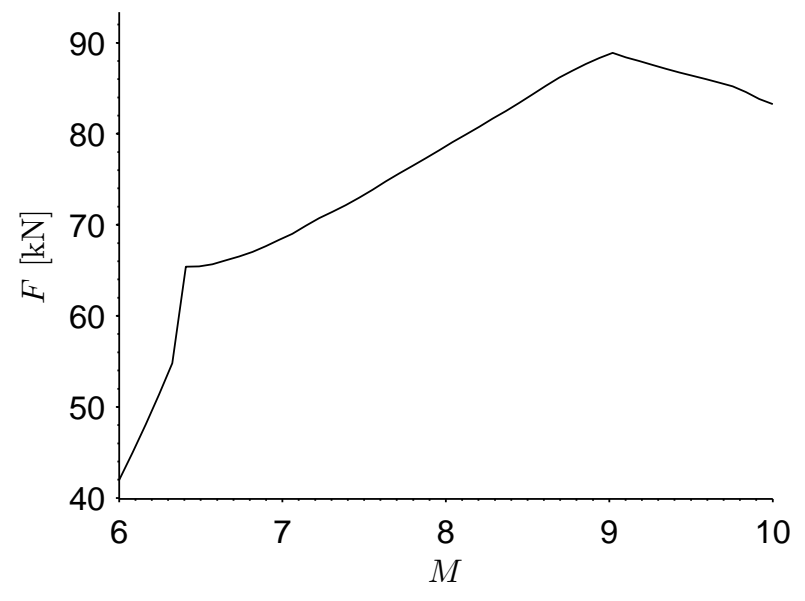

b) Thrust vs Mach number for the baseline inlet design of Fig. 3, in N.

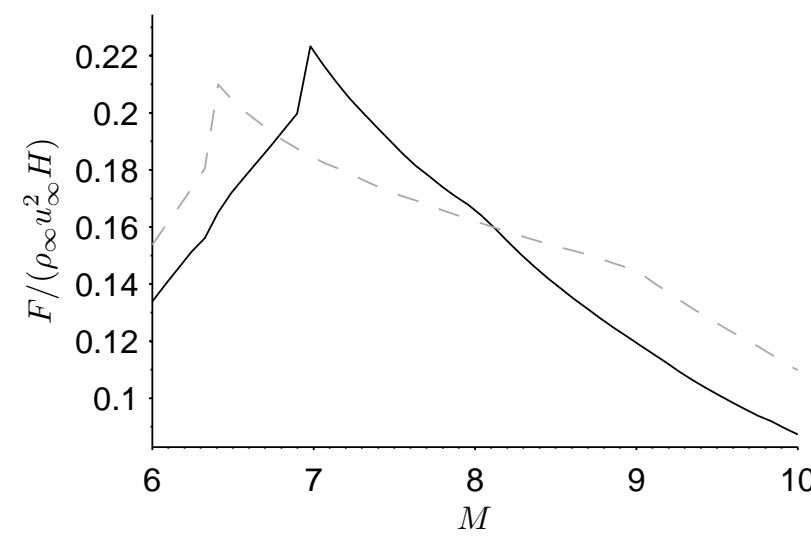

c) Thrust vs. Mach number for the simple and baseline designs, normalized by captured momentum, $\rho u^{2} H_{\text {inlet }}$. The solid line is the simple flowpath performance and the dashed line is the baseline flowpath performance.

Figure 2. Comparison of the performance of the simple design of Fig. 1 to the performance of the baseline design, shown in Fig. 3.

$$
3 \text { of } 14
$$




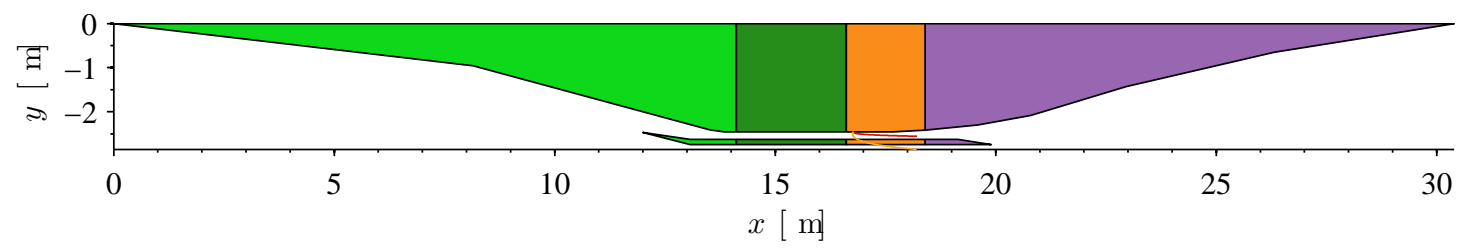

Figure 3. Baseline vehicle design, which has performance that does not change abruptly for off-design conditions.

of the duct. This physical process leads to the creation of a Pre-Combustion Shock Train (PCST), which in turn allows the dual-mode flowpath to operate both sub- and supersonically. The following is a brief discussion of how steady, 1-D flows can be solved in a way that allows for choking conditions to be considered.

\section{A. Ram-mode operation}

Previous authors ${ }^{7,8}$ have proposed a method to solve the ram-scram problem. Heiser and Pratt ${ }^{7}$ rely on the information presented in Shapiro's ${ }^{8}$ book for the case of heat release in a diverging duct. They assume that the gas has constant specific heats and molecular weight such that the Mach number is well-defined. They conclude that if the flow is thermally choked (ram mode) choking occurs where the following equation has a minimum.

$$
\left(\frac{1}{A_{\mathrm{eff}}} \frac{d A_{\mathrm{eff}}}{d x}\right) \equiv\left(\frac{1}{A} \frac{d A}{d x}\right)-\frac{\left(1+\gamma M^{2}\right)}{2}\left(\frac{1}{T_{0}} \frac{d T_{0}}{d x}\right)
$$

The cross-sectional area of the duct is $A, x$ is the downstream axial coordinate, $M$ is the Mach number, $\gamma$ is the ratio of specific heats, $T_{0}$ is the stagnation temperature and $A_{\text {eff }}$ is the effective cross-sectional area. By effective cross-sectional area, Heiser and Pratt mean that we can use this definition analagously to the physical area in an isentropic flow. However, as they note, the solution of this equation for the minimum point only gives a point at which a transonic condition can occur-it does not say whether one will occur.

Mach number is not used in MASIV. However, it is convenient to use the definition of Mach number $M=u / \sqrt{\gamma p / \rho}$ in this step because it accurately predicts the location of the singularity corresponding to $M=1$ in the set of ODEs. If conditions can be found which cause the effective area to have the right properties then the Mach number will proceed through unity at the singularity.

Enter the forcing function. Shapiro proposes a function called $G$ :

$$
\begin{aligned}
\frac{1}{M^{2}} \frac{d M^{2}}{d x} & =\frac{G(x)}{1-M^{2}} \\
G(x) & \equiv G\left(M, A(x), T_{0}(x), \gamma, C_{f}, D, \ldots\right)
\end{aligned}
$$

Numerous authors show how (2) can be derived from the conservation equations. The definition of the $G$-function is simply the forcing term from the right-hand side of the Mach number equation for 1-D flow. For example, for 1-D flow with heat addition and area change (but no friction or mass addition), the Mach number equation is ${ }^{8}$

$$
\frac{1}{M^{2}} \frac{d M^{2}}{d x}=-\frac{2\left(1+\frac{\gamma-1}{2} M^{2}\right)}{1-M^{2}} \frac{1}{A} \frac{d A}{d x}+\frac{\left(1+\gamma M^{2}\right)\left(1+\frac{\gamma-1}{2} M^{2}\right)}{\left(1-M^{2}\right)} \frac{1}{T_{0}} \frac{d T_{0}}{d x}
$$


and so $G$ is

$$
G=-2\left(1+\frac{\gamma-1}{2} M^{2}\right) \frac{1}{A} \frac{d A}{d x}+\left(1+\gamma M^{2}\right)\left(1+\frac{\gamma-1}{2} M^{2}\right) \frac{1}{T_{0}} \frac{d T_{0}}{d x}
$$

The function is more complex when other effects are added. In MASIV this predictive approach is not viable since we use total enthalpy and velocity instead of Mach number and stagnation temperature. However, the principle is the same and $G$ can be defined in terms of primitive variables:

$$
G=\left(1-\frac{\rho u}{\gamma p}\right)\left(\frac{1}{u^{2}} \frac{d u^{2}}{d x}+\frac{1}{\rho} \frac{d \rho}{d x}-\frac{1}{p} \frac{d p}{d x}\right)
$$

Next we consider some cases. For hypersonic engine flowpaths we only consider supersonic conditions at the entrance to the isolator/combustor section and supersonic outflow conditions at the exit of the nozzle. The case of $1<M<\infty$ corresponds to pure scram and can be solved by integration of the ODEs in MASIV; no further comment is required. This leaves the case where $M=1$ at least once in the isolator/combustor section.

We are interested only in flows that transition smoothly from subsonic to supersonic or from supersonic to subsonic. In general the latter are not stable under small perturbations, ${ }^{9}$ so we discard this case immediately. This implies the first necessary condition:

1. $M$ must change from $M<1$ to $M>1$ with increasing $x$ on the interval of interest.

If $M$ is equal to unity more than once the interval may be subdivided into intervals on which $M=1$ exactly once, so we only consider the case in which $M=1$ exactly once. Most reasonable dual-mode combustors have only one choking location.

Because of the requirement that $M=1$ at exactly one point (due to thermal choking) it is possible to identify constraints on the $G$-function that are necessary for smooth transition through the sonic point. Shapiro provides these on $\mathrm{p} .256 .{ }^{8}$ The particular case of interest for the present study is Case V: $G$ Changes from Positive to Negative. This provides the second necessary constraint, which is that

2. $G$ must change from positive to negative where $M=1$.

Any region satisfying these two constraints will transition smoothly from subsonic to supersonic.

\section{Methodology}

Once the constraints on the solution are known, the difficulty of how to satisfy the constraints remains. One method for dealing with this difficulty is to use a shooting method. One simply searches for initial conditions that cause the Mach number to equal unity precisely when $G=$ 0 . The solution that meets this requirement is the allowed ram solution for the given boundary conditions at the beginning of the isolator (which must be supersonic).

The algorithm is as follows.

1. The inlet solution (from the SAMURI code) provides a supersonic upstream boundary condition for the isolator. 
2. First assume that the flow will remain supersonic throughout the isolator and combustor. Attempt to compute a scram solution. If solution succeeds, then the engine operates in scram mode. No pre-combustion shock train is required. End.

3. If the sonic point was approached at any $x$-location then this flow does not have a stable supersonic solution. Determine the ram-mode solution.

Once a ram case has been identified, the solution proceeds according to Shapiro's method, outlined above. However, a difficulty arises because in general $G$ is a function of the boundary conditions and the state variables so it cannot be computed ahead of time. Hence, it is impossible to identify the choking location without performing some type of iterative solution. A backstepping search method was used because of its robustness. The iterative procedure follows.

1. Assume that there is a PCST in the isolator which sets conditions at the beginning of the combustor. The PCST is of unknown strength. Bracket the condition which allows a transonic solution by assuming

$$
\begin{aligned}
& M_{3}^{i=1} \approx 0.1 \\
& M_{3}^{i=2}=\sqrt{\frac{1+\frac{\gamma-1}{2} M_{2}^{2}}{\gamma M_{2}^{2}-\frac{\gamma-1}{2}}}
\end{aligned}
$$

where the upper bound (8) is set by the single normal shock solution.

2. Compute flow solution throughout the duct until a sonic condition is reached, or the solution indicates that the flow is subsonic everywhere. If point 1 does choke and point 2 does choke, then this is a valid starting point; proceed onward. If not, iteration points 1 and 2 do not bracket a valid starting point; return to step 1 and multiply the lower bound $\left(M_{3}^{i=1}\right)$ by $\rho<1$.

3. Perform a binary search for the interface between choking/non-choking inital condition. Terminate when $\left|\left(M_{3}\right)^{i+1}-\left(M_{3}\right)^{i}\right| /\left(M_{3}\right)^{i}<\varepsilon_{r}$, where $\varepsilon_{r}$ is the relative error tolerance $\approx 10^{-6}$. Let $x_{C}=x^{i}$, which is the predicted location of choking.

4. Test the $G$-function at $M_{3}$. If $|G|<\varepsilon_{g}$, terminate the search successfully and proceed. If $G$ is not close to 0 , this is not a valid solution.

5. Now compute the Mach number downstream of the thermal choking location. Estimate the new state by using the shock-jump relations for $M, p, T$. When the initial Mach number in the jump equation is less than unity, entropy is destroyed. However, the error incurred in this step can be made as small as required by setting tolerance $\varepsilon_{r}$.

6. Re-start combustor solution at $x_{C}+\Delta$, where $\Delta$ is some small step past the singularity.

\section{A. Isolator solution}

Numerous authors ${ }^{7,10}$ indicate that the isolator can be approximated by separated regions near the walls and a compressible, inviscid core flow. This in turn implies that in the core region the only allowable decrease in flow stagnation pressure is that due to waves. The stagnation temperature and other flow properties, such as ratio of specific heats, $\gamma$, viscosity, etc. are assumed not to vary. 
The equation relating $p_{3} / p_{2}$ and $M_{3} / M_{2}$ is given by Heiser and Pratt. ${ }^{7}$

$$
\frac{p_{3}}{p_{2}}=1+\gamma^{2} M_{2}^{2}-\gamma M_{2} M_{3} \sqrt{\frac{1+\frac{\gamma-1}{2} M_{2}^{2}}{1+\frac{\gamma-1}{2} M_{3}^{2}}}
$$

The stagnation enthalpy in the core flow does not vary, which gives the temperature equation.

$$
\frac{T_{3}}{T_{2}}=\frac{1+\frac{\gamma-1}{2} M_{2}^{2}}{1+\frac{\gamma-1}{2} M_{3}^{2}}
$$

The inlet code (SAMURI) computes $M_{2}, T_{2}$, and $p_{2}$ at the entrance to the isolator. The method of the previous section provides $M_{3}$ and $p_{3}$ by satisfying the $M=1$ constraint due to choking in the combustor. Ikui ${ }^{10}$ proposes a method for estimating the pressure rise as a function of $x$ in the isolator. We used this method, but replaced the mixing boundary condition with the requirement that $\frac{d p}{d x}=0$ at the end of the PCST. This gives the following equations.

$$
\begin{aligned}
w & =\frac{M}{\frac{2}{\gamma-1}\left(1+\frac{\gamma-1}{2} M^{2}\right)} \\
w^{\prime}(x) & =w_{3} \cosh \{b(l-x)\} \\
\frac{p(x)-p_{2}}{p_{3}-p_{2}} & =\frac{w_{2}-w^{\prime}(x)}{w_{2}-w_{3}}
\end{aligned}
$$

Equation 11 defines the Crocco number $w, w^{\prime}(x)$ is the Crocco number between station 2 and station $3, b$ is an experimentally determined constant and $p(x)$ is the static pressure in the isolator between $p_{2}$ and $p_{3}$. The estimated length of the PCST, $l$, is determined by using Ikui's experimental correlation, $l=\frac{1}{c} \ln \left(w_{2} / w_{3}\right)$, where $c$ is also experimentally determined. The temperature is computed using Eq. (10). Thus, the thermodynamic solution is computed throughout the isolator and combustor. The ratio of specific heats $\gamma$ is assumed not to vary in the isolator. An example solution that includes choking in the combustor is shown in Fig. 4. Note the smooth pressure rise in the isolator and the steepness of $\frac{d M}{d x}$ around $M=1$.

\section{Performance of available designs}

In order to obtain a starting point for the design trade study, we begin with some designs that are available in the open literature. ${ }^{2,11}$ These are laboratory scale combustors which are used for tests of combustion processes such as flame holding and combustion efficiency. It is important to note that these combustors are not necessarily designed to generate thrust, but they do provide a reasonable starting point for combustor design because they are combustors known to operate successfully. The referenced authors and the designers of these combustors have made no claims about any positive or negative performance qualities they may have. Furthermore, the configurations used in the experiments cited were not able to be modeled without some modifications that allowed them to fit into the design structure that MASIV uses.

\section{A. Description of existing designs}

Figure 5 shows the geometry of the combustor used by Micka et al. ${ }^{2}$ Thrust information is not available for this model. Pressure measurements throughout the isolator and combustor, Schlieren 


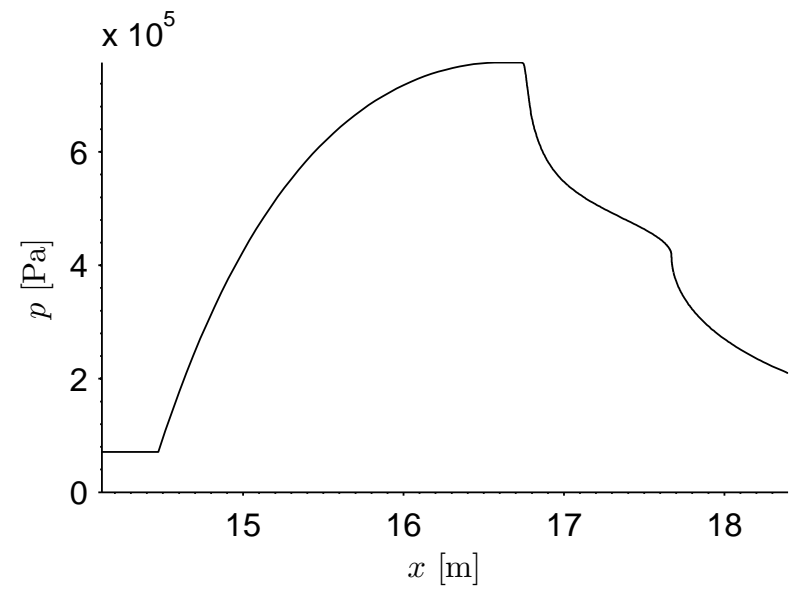

a) Pressure solution

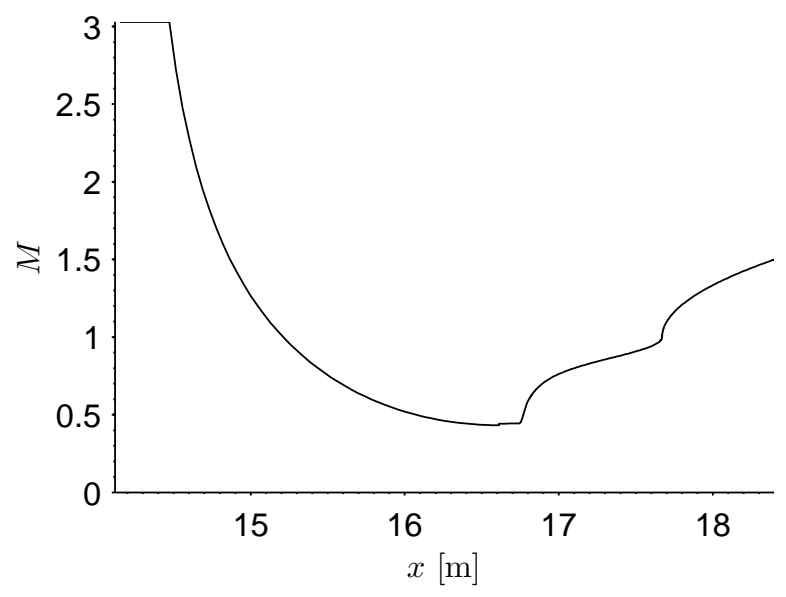

b) Mach number solution

Figure 4. An example of a ram-mode solution as computed by MASIV. 
images of the PCST, chemiluminescence images of the reaction region and planar lasar-induced fluorescence (PLIF) images of the reaction region are available.

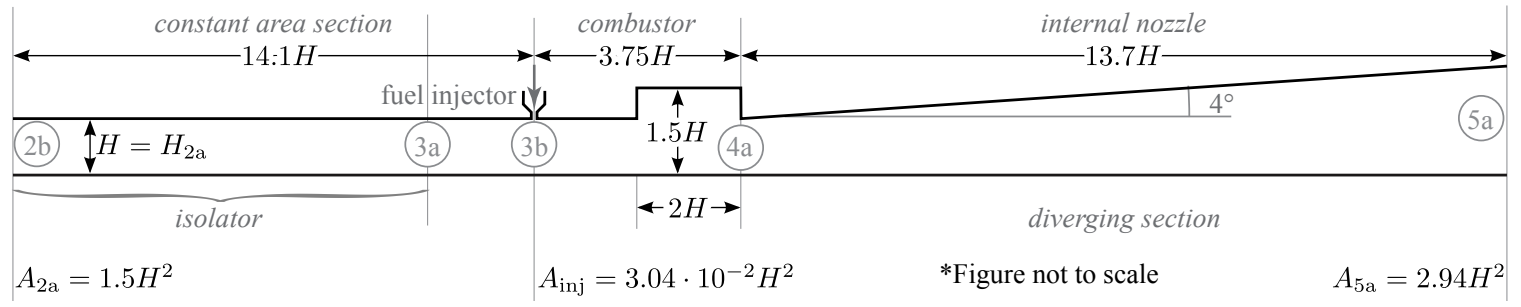

Figure 5. The combustor configuration of Micka et al. .2

Figure 6 shows the geometry of the combustor used by Donde et al., ${ }^{11}$ which originates with Dr. James Donohue at United Technologies Research Corporation (UTRC). A data set for this combustor is not yet available.

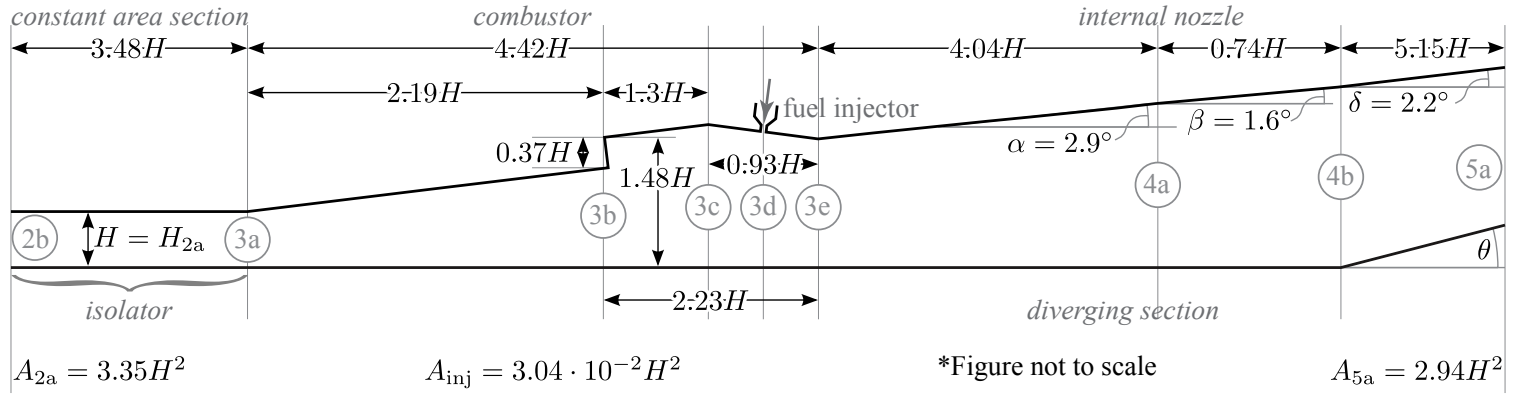

Figure 6. The combustor configuration described by Donde et al. ${ }^{11}$

These test combustors are basically appropriate to be analyzed using MASIV, however, a few extra assumptions and modifications need to be made. All the combustors have fairly low-aspectratio cross-sections. In general the vehicles assembled automatically with MASIV have highaspect-ratio cross-sections. This is because making the engine deeper (into the page) allows more mass flow and hence more thrust without a large vehicle drag penalty; the drag of the flowpath per unit depth remains constant.

We assume that the spacing of injectors of each of the combustors is the same although different injector spacing, injector diameter and total injector area were used in each experiment. This injector sizing and spacing is determined by jet penetration requirements at the design equivalence ratio. The experiments in question had low equivalence ratios $(\phi \approx 0.25$ in the Micka experiment and $\phi \approx 0.08$ in the Donohue experiment), which allowed for small-diameter injectors for good penetration. With a higher equivalence ratio called for due to thrust requirements, a larger injector size and higher injector pressure makes sense. This aspect of the design can and should be optimized.

Injection location had to be modified somewhat, due to the fact that the MASIV code cannot simulate stagnant flows, vortical structures, or reversed flow, all of which one would encounter in a typical cavity. Hence, cavity injection is not considered in the present analysis. For the Micka experiment this is not a major concern since between $95 \%$ and $100 \%$ of the fuel was injected through wall injector and only a small pilot fuel supply is used to ensure that the flame ignites. In the Donohue experiment, fueling was through one of the cavity walls. This fueling configuration 
would probably not achieve significant penetration into the flow for higher equivalence ratios, and MASIV cannot simulate it. The cavity fueling is replaced with a set of wall-normal injectors at the same location.

The wall geometries of the experiments are reproduced exactly in the MASIV geometries, except for scaling and the removal of the wall cavities, as discussed above. In practice this is not a serious limitation because the wall cavities have little effect on the area profile seen by the core flow. If desired, the cavity can be simulated by using an area profile that follows the streamline connecting the leading and trailing edges of the cavity. Table 1 explains the differences between the configurations reported in the experiments and those used in MASIV.

Table 1. Geometric configurations from experiments and MASIV

\begin{tabular}{|c|c|c|c|c|c|}
\hline & & \multicolumn{2}{|c|}{ Experiment actual } & \multicolumn{2}{|c|}{ MASIV version } \\
\hline & & Micka & Donohue & Micka & Donohue \\
\hline \multirow{6}{*}{$\begin{array}{l}\overparen{E} \\
\mathbb{0} \\
0 \\
0 \\
0 \\
\tilde{U} \\
\tilde{0}\end{array}$} & Cavity angle & $90^{\circ}$ & $21.8^{\circ}$ & N/A & N/A \\
\hline & Cavity depth & $0.5 H$ & $0.37 H$ & N/A & N/A \\
\hline & Cavity length & $2 H$ & $2.23 H$ & N/A & N/A \\
\hline & $\mathrm{x}$-section $R$ & 1.5 & 3.35 & 12.9 & 12.9 \\
\hline & $\mathrm{N}$ divergences & 1 & 3 & 1 & 3 \\
\hline & $\frac{H_{5}}{H_{2}}$ & 1.24 & 1.65 & 1.24 & 1.65 \\
\hline \multirow{6}{*}{$\begin{array}{l}\dot{\Xi} \\
\dot{0} \\
0 \\
\dot{\Xi}\end{array}$} & $\mathrm{x}$-location ${ }^{\dagger}$ & $-3.75 H$ & $3.96 H$ & $-5.37 H$ & $0 H$ \\
\hline & $x$-location ${ }^{\ddagger}$ & $14.1 H$ & $7.44 H$ & $16 H$ & $16 H$ \\
\hline & $\mathrm{N}$ injectors & 1 & 13 & 25 & 25 \\
\hline & $n=N /$ depth & $0.67 \mathrm{H}^{-1}$ & $3.88 \mathrm{H}^{-1}$ & $1.94 \mathrm{H}^{-1}$ & $1.94 \mathrm{H}^{-1}$ \\
\hline & $\mathrm{d}$ injectors & $0.098 H$ & $0.018 H$ & $0.22 H$ & $0.22 H$ \\
\hline & A injectors & $7.6 \cdot 10^{-3} H^{2}$ & $3.2 \cdot 10^{-3} H^{2}$ & $0.99 H^{2}$ & $0.99 H^{2}$ \\
\hline
\end{tabular}

$\dagger$ measured downstream from first divergence

$\ddagger$ measured downstream from beginning of isolator

\section{B. Design-space analysis}

The engine flowpaths were integrated into an $\mathrm{x}$-43-like hypersonic vehicle that is described by Torrez et $a l^{3}$ The designs have all been analyzed over the same range of conditions inside of the same vehicle design, insofar as this was possible. Of course, since the designs have different lengths, and different heights from beginning to end, some assumptions had to be made. The different lengths of combustor required slightly different vehicle lengths, but the overall inlet, isolator and nozzle shapes were kept the same between the flowpath geometries. The nozzles varied slightly in their combustor attachment locations due to the differences in combustor geometry.

The desired operating range was defined to be $6<M<8$ and $18000 \mathrm{~m}<$ altitude $<28000 \mathrm{~m}$. All the flowpaths presented have the same mass flow condition at a given operating point, so the thrust curves generated by the flowpaths can be compared. Angle of attack was $\alpha=0^{\circ}$ and equivalence ratio was $\phi=0.5$ for all operating maps. 


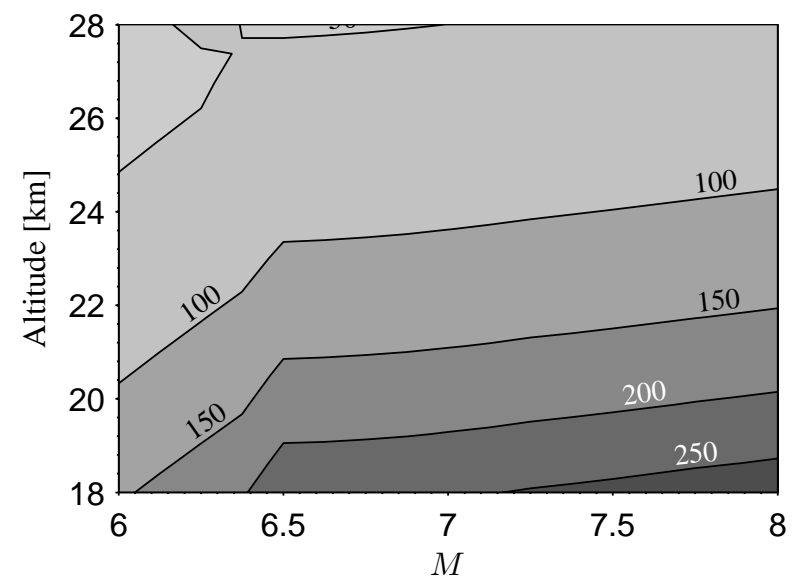

Figure 7. Operating map for the flowpath geometry shown in Fig. 5. Curves represent different values of thrust in $\mathrm{kN}$.

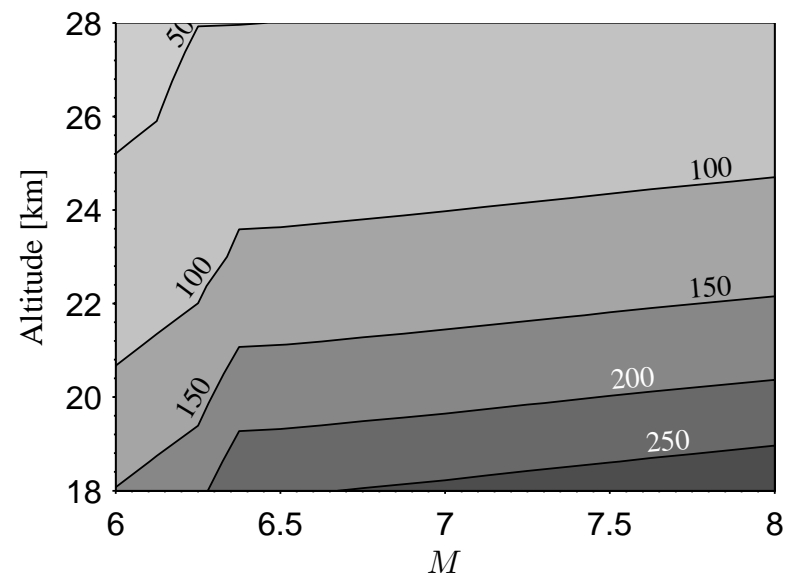

Figure 8. Operating map for the geometry shown in Fig. 6, but with injectors upstream of the first divergence by $H$. Curves represent different values of thrust in $\mathrm{kN}$. 


\section{Results}

Comparing Fig. 7 to Fig. 8 several characteristics can be noted. Let us begin with the obvious kink in the operating maps where the isocontours change slope. This occurs at about $M=6.5$ in Fig. 7 and about $M=6.3$ in Fig. 8. This kink is due to a mode transition in the combustor. In ram mode (at the lower Mach numbers) less thrust is produced than in scram mode at the same altitude. The PCST present in the isolator in ram mode causes stagnation pressure loss. No PCST exists in scram mode operation, so the thrust generated is larger. The mode transition happens when too much fuel is added to the supersonic flow and it chokes.

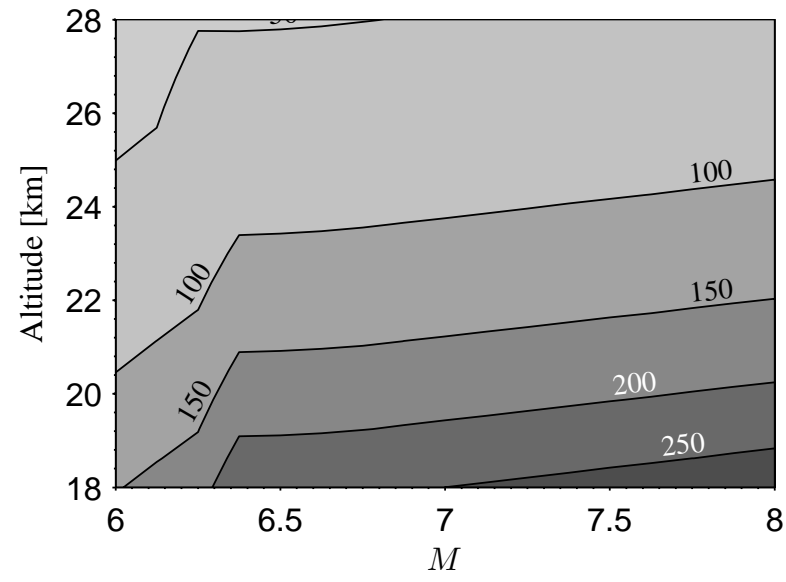

Figure 9. Operating map for the flowpath geometry shown in Fig. 5, but with injectors moved farther upstream by $2 H$. Curves represent different values of thrust in $\mathrm{kN}$.

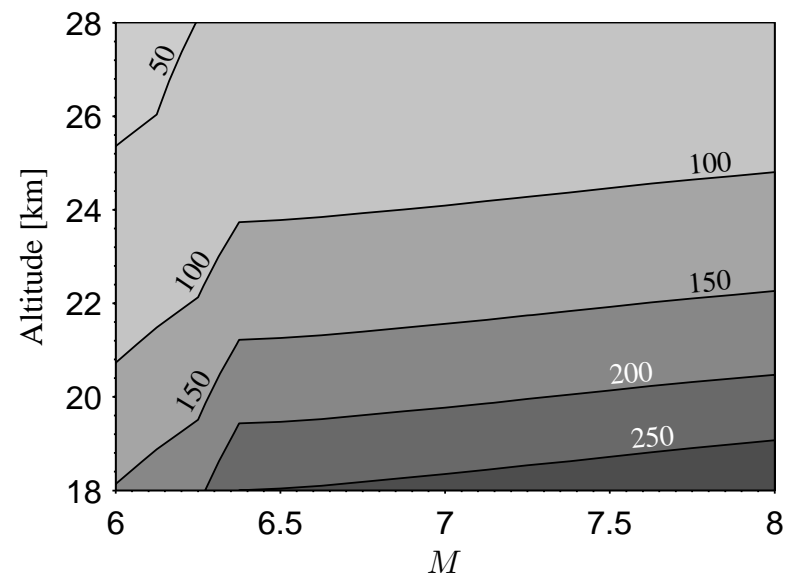

Figure 10. Operating map for the geometry shown in Fig. 6, but with injectors upstream of the first divergence by $3 H$. Curves represent different values of thrust in $\mathrm{kN}$.

If we concentrate on the total thrust, it is clear that the Donohue combustor flowpath produces more thrust for all altitudes and flight Mach numbers considered than the Micka combustor flowpath. This is attributed to the larger geometric expansion of the flow in the combustor before it enters the nozzle.

Returning to the kink in the performance maps, it seems the the increased performance of the 


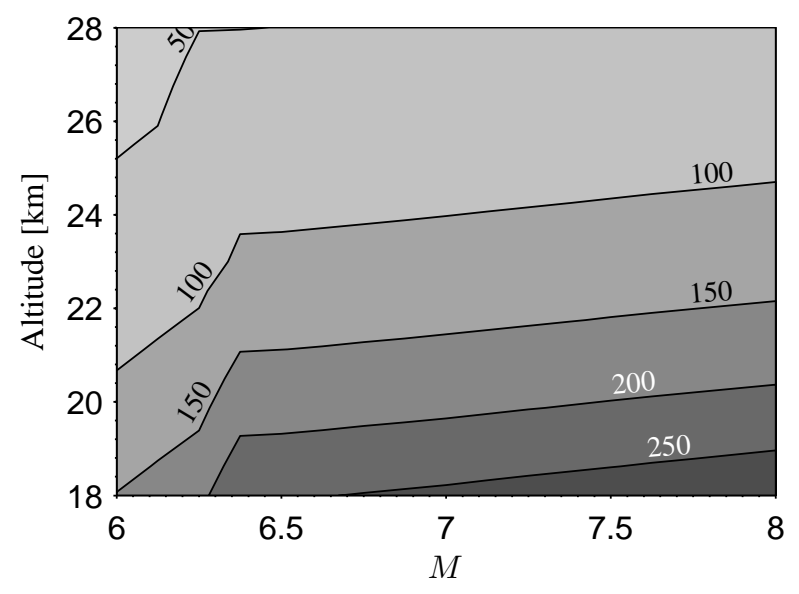

Figure 11. Operating map for the geometry shown in Fig. 6, but with injectors located at the first divergence (marked station 3a). Curves represent different values of thrust in $\mathrm{kN}$.

Donohue-type combustor comes at a price. The slope of the isocontours around the kink is much larger in Fig. 8 than in Fig. 7. The greater the change in thrust due to mode transition, the greater a control problem a mode transition may cause. In these examples it appears that an extremely rapid change in thrust produced will occur if the Mach number decreases or the altitude increases as fueling remains constant ( $\phi$ is fixed), triggering a mode transition.

If the injectors are moved farther upstream so that more of the flame length is inside the constant-area section, Fig. 9 and Fig. 10 show that slightly more thrust is produced by both flowpaths, and the slope of the ram-mode to scram-mode transition point for both may be slightly reduced. A grid-refinement study is required to understand what the true slope is at this point, since it is possible for the location of points to obscure the slope of this transition, or the existence of a discontinuity at this point.

Conversely, Fig. 11 shows that if the injectors are moved downstream to coincide with the first divergence, the thrust is reduced in both ram mode and scram mode and the sensitivity to mode transition increased slightly. No results are presented for the Micka flowpath with injectors coincident with the first divergence because this configuration has no ram-mode solution. This is predicted by the fact that there is no way for the heat release in the diverging section to be balanced out by area change as predicted by Eq. 1 if the heat release and area change profiles are not compatible. It is easier for this balance to occur in the Donohue combustor because of the varying $\frac{d A}{d x}$.

\section{Conclusions}

This paper presents a method of analyzing dual-mode hypersonic engine flowpaths. Combined with previous work, the method allows consideration of choking in flowpaths that have the right combination of operating point and fuel addition. Two flowpath configurations from the literature were presented and were modeled with some modifications using the MASIV code. Several variations of each combustor were presented, based on moving the location of fuel injectors relative to the diverging section of each flowpath.

Based on these results, it seems that flowpath divergence location and angle as well as injector 
location are good candidates for optimization using a multidisciplinary optimization tool.

\section{References}

${ }^{1}$ Mathur, T., Gruber, M., Jackson, K., Donbar, J., Donaldson, W., Jackson, T., and Billig, F., "Supersonic Combustion Experiments with a Cavity-Based Fuel Injector," Journal of Propulsion and Power, Vol. 17, No. 6, NovemberDecember 2001.

${ }^{2}$ Micka, D. J. and Driscoll, J. F., "Combustion characteristics of a dual-mode scramjet combustor with cavity flameholder," Proceedings of the Combustion Institute, Vol. 32, 2009, pp. 2397-2404.

${ }^{3}$ Torrez, S. M., Driscoll, J. F., Dalle, D. J., and Fotia, M. L., "Preliminary Design Methodology for Hypersonic Engine Flowpaths," 16th AIAA/DLR/DGLR International Space Planes and Hypersonic Systems and Technologies Conference, 2009.

${ }^{4}$ Torrez, S. M., Driscoll, J. F., Bolender, M. A., Oppenheimer, M. W., and Doman, D. B., "Effects of Improved Propulsion Modelling on the Flight Dynamics of Hypersonic Vehicles," AIAA Atmospheric Flight Mechanics Conference and Exhibit, 2008, AIAA Paper No. 2008-6386.

${ }^{5}$ Torrez, S. M., Driscoll, J. F., Ihme, M., and Fotia, M. L., "Reduced Order Modeling of Turbulent Reacting Flows With Application to Scramjets," Journal of Propulsion and Power, Vol. 27, No. 2, March-April 2011, pp. 371-382.

${ }^{6}$ O'Brien, T. F., Starkey, R. P., and Lewis, M. J., "Quasi-One-Dimensional High-Speed Engine Model with Finite-Rate Chemistry," Journal of Propulsion and Power, Vol. 17, No. 6, 2001, pp. 1366-1374.

${ }^{7}$ Heiser, W. H. and Pratt, D. T., Hypersonic Airbreathing Propulsion, AIAA Pub., Washington, DC, 1994.

${ }^{8}$ Shapiro, A. H., Dynamics and Thermodynamics of Compressible Fluid Flow, Ronald Press, NY, 1953.

${ }^{9}$ Kerrebrock, J. L., Aircraft Engines and Gas Turbines, The MIT Press, 2nd ed., 1992.

${ }^{10}$ Ikui, T., Matsuo, K., and Nagai, M., "The Mechanism of Pseudo-Shock Waves," Bulletin of the JSME, Vol. 17, No. 108, June 1974, pp. 731-739.

${ }^{11}$ Donde, P., Koo, H., and Raman, V., "A Multivariate Quadrature Based Moment Method for Supersonic Combustion Modeling," 49th AIAA Aerospace Sciences Meeting including the New Horizons Forum and Aerospace Exposition, January 2011, AIAA 2011-322. 